评 述 中国科学院 科学与技术前沿论坛 合成生物学专辑

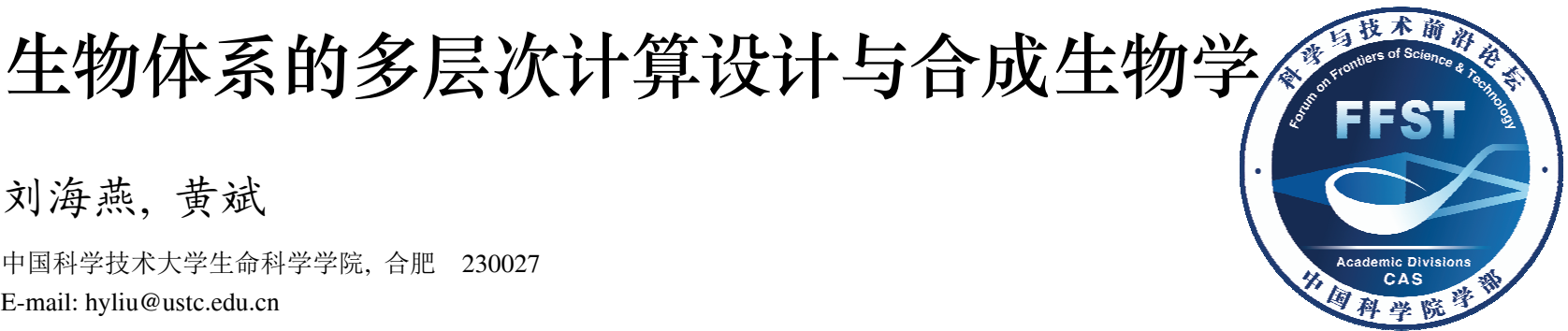

收稿日期: 2015-03-16; 接受日期: 2015-05-20

国家重点基础研究发展计划(批准号：2011CBA00803)、国家自然科学基金委员会-中国科学院学科发展战略研究合作项目(批准号: L1222037)和中国科学院合肥大科学中心研究项目 (批准号: 2015SRG-HSC041)资助 doi: 10.1360/N052014-00261

摘要 合成生物学的核心思想是将现代工程学的原理与方法引入对生命系统的改造和 构建中. 生命活动覆盖从分子到细胞再到有机体等不同层次. 合成生物学研究同样跨越 了多个层次, 例如, 在分子层次进行生物元件和器件的设计和标准化、通过合成基因线路 研究生物网络的设计和调控原理、在途径和网络层次进行细胞内代谢网络和代谢途径的 人工设计改造等. 本文一方面试图对与此有关的既有计算机模拟与设计方法加以总结和

\section{关键词}

生物信息学方法

分子设计

网络动力学

代谢网络分析 介绍，另一方面探讨这些不同层次的计算模拟与设计工具可应用于哪些方面的合成生物 学问题, 以及既有方法可能在哪些方向上还有比较大的发展潜力, 能更好满足合成生物 学研究需求.

合成生物学中的计算设计是不同层次计算生物 学方法的综合应用. 在分子层次, 从天然生物分子中 发掘可用元器件需要用到序列结构比较、化学相似性 比较、结构和功能预测等计算工具; 进化设计、基于 结构的设计等可以应用于以天然生物分子为模板进 行元器件功能的改造、优化, 甚至引入新的功能. 在 网络层次, 基因线路等具有信息处理、调控能力的模 块的设计需要建立网络动力学模型并对其进行分析、 预测; 流量平衡分析可用于预测代谢网络在经过人 工改造前后的代谢能力; 基于代谢反应数据库的代 谢路径检索、发现软件可以为代谢途径设计提供指导. 本文将对这些计算方法所针对的问题及其基本原理 做概要的介绍, 另外还简要介绍了本实验室在这方 面的几项相关工作.

\section{1 分子层次的计算与设计}

\section{1 生物分子的序列和结构比较}

序列/结构比较的目的是判断不同生物大分子序 列和结构之间是否存在相似性, 并在此基础上建立 起不同序列上的氨基酸位置(或核苷酸位置)之间的 对应关系(称为 alignment) ${ }^{[1 \sim 3]}$. 基于序列/结构比较, 可以根据对其他生物分子的既有知识对研究者们所 关心的生物分子及其某些方面的特性做出预测, 并 给出能反映预测可信程度的统计参量. 此外, 还可以 基于不同生物分子的相似和不同之处对序列-结构功能关系进行总结.

（1）整体比较. 目前, 在进化同源性基础上的 蛋白质和核酸序列比对方法如动态规划 ${ }^{[1,2]} 、 \mathrm{BLAST}^{[4]}$ 等已非常成熟, 被广泛应用于同源性判别、数据库查

引用格式: 刘海燕, 黄斌. 生物体系的多层次计算设计与合成生物学. 中国科学: 生命科学, 2015, 45: 943-949 Liu H Y, Huang B. Computation and design of biological systems at different levels in synthetic biology. SCIENTIA SINICA Vitae, 2015, 45: 943-949, doi: 10.1360/N052014-00261 
询、多序列的进化关系分析 ${ }^{[5]}$ 等. 对蛋白质而言, 在 其进化过程中空间结构的保守性可能远高于其氨基 酸序列的序列保守性. 因此, 距离矩阵比对 (alignment of distance matrices, Dali) ${ }^{[3]}$ 、组合扩展 (combinatorial expansion, $\mathrm{CE})^{[6]}$ 等蛋白质空间结构比 较与比对方法可用于识别进化上非常远缘、序列相似 度很低的同源蛋白质, 也可以用来建立同源序列之 间更精确的氨基酸位置对应关系.

上述经典的序列/结构比较方法建立在被比较的 生物大分子的序列/结构在整体上(或至少在结构域层 次上)存在相似性的基础之上. 整体序列或结构的相 似性并不是生物信息学对分子的结构/功能特性进行 预测或设计所必需的. 在整体相似性不存在或不明 显时，局部比较仍有可能提供有用信息.

（2）局部序列比较. 局部特征如序列 motif 对决 定某些功能特性(例如, 核酸或蛋白酶的识别位点、转 录因子识别位点、蛋白质定位信号、蛋白质修饰位点、 蛋白质与一些特殊配体基团如核苷、特定金属离子等 的结合位点、某些特殊超的二级结构如 coiled-coils 等 $)^{[7 \sim 9]}$. 相对简单的序列 motif 可通过人工经验总结 得到. 针对不同问题的生物信息学研究也积累了大 量的序列 motif 及其发现和应用算法, 包括相关数据 库等, 其中大部分作为互联网上的公共资源对研究 者开放.

（3）局部结构比较. 基于空间结构对生物大分 子进行局部比较/比对的研究目前还不太多. 其中, 蛋白质配基结合位点比较方法(structural comparison of protein ligand binding sites, SMAP)能够基于活性位 点(配体或底物结合位点)周围氨基酸残基的空间排 列对蛋白质空间结构进行不依赖于一级序列顺序的 两两比较, 并进行显著性检验 ${ }^{[10]}$. 采用 SMAP 或类似 方法能够对进化上更加远缘、甚至非同源而只是通过 所谓的“趋同进化”(convergent evolution)而得的具有 相同或相近功能的活性位点进行识别和分析, 相对 于用近同源分子进行分析比对, 更有可能总结出序 列-结构-功能之间的因果联系.

（4）化学相似性比较. 代谢中间物等有机小分 子作为底物或调节物与蛋白质、核酸等大分子的相互 作用在合成生物学中占据重要地位. 尽管组成一般 有机分子的化学结构单元并不限于有限的氨基酸或 核苷酸类型，不同分子之间也不存在进化上的同源 性, 不同分子的功能仍有可能通过其化学结构/空间
结构的相似性建立关联. 近年来, 基于化学指纹编码 或公有子化学结构等对一般有机分子进行相似性比 较、基于相似性进行数据库查询等工具逐步走向成熟 和实用化 ${ }^{[11 ~ 13]}$. 人工代谢途径设计、小分子与大分子 人工相互作用设计等是合成生物学的重要课题, 化 学信息学及其与生物信息学的结合可能在基于数据 进行这类设计方面取得重要进展.

\section{2 生物分子结构和功能预测}

（1）结构预测. 测定生物大分子的序列已变得 非常容易, 但空间结构测定还是非常耗时耗力. 相对 于序列, 空间结构往往包含更加明确、可靠的功能信 息. 因此, 长期以来, 结构预测一直是计算生物学的 重要任务. 从蛋白质结构预测竞赛 CASP(critical assessment of protein structure prediction)开展的十多 年来, 蛋白质三维结构的从头预测取得了非常大的 发展 ${ }^{[14]}$, 在某些情况下已可用做蛋白质结构解析的 辅助工具. 另一方面, 在纯粹从预测得到的空间结构 模型来考虑, 比较建模(comparative modeling)是最可 靠和精度最高的 ${ }^{[15]}$, 但比较建模建立在同源空间结 构模板已存在且能被正确识别(如通过序列比对)基 础之上, 其“发现”的意义并不明显. 对功能研究而言, 基于折叠识别的结构预测方法如“threading”的意义 可能更大 ${ }^{[16,17]}$. 已有部分研究实例表明, 通过 “threading”, 有可能发现不能通过序列水平的比较被 发现或确认的远缘同源关系, 并基于此对未知蛋白 的功能做出预测. 相对蛋白质结构预测而言, 核酸 (主要是 RNA)的结构预测(主要是二级结构预测)要简 单一些 ${ }^{[18]}$. 由于 mRNA(包括未翻译区 (untranslated region, UTR))的二级结构是影响基因表达的重要因 素, mfold 等 RNA 二级结构预测程序是基因设计中的 常用工具 ${ }^{[19]}$.

(2) 功能预测. 组学分析能够高通量地发现新 的基因、蛋白质、非编码 RNA 等生物分子及其可能 的突变体, 下一步生物功能分析与整合的起点往往 是对它们进行功能预测. 功能预测使用最广泛 (也往 往最有效)的策略是基于同源性或相似性：根据目标 分子与(数据库)中已知分子在某些特定方面的相似/ 差异程度, 预测目标分子是否可能具有已知分子的 某种特性或功能. 因此, 功能预测实际上是前述各类 序列/结构比较和比对方法的应用. $\mathrm{BLAST}^{[4]}$, $\mathrm{FASTA}^{[20]}$, Dali ${ }^{[3]}$ 等作为基本工具被广泛用于在通用 
序列和结构数据库中检索是否有与目标蛋白质或核 酸分子同源的已知分子, 进而能获取目标分子相关 的既有功能研究结果; 通过一些专门数据库可分析 目标分子是否包含已知的、与特定功能联系的局部序 列 motif, 从而预测目标分子特性; 如果采用这些可 靠性较高、但灵敏度不足的方法未能获得足够多的信 息, 还可以使用一些灵敏度更高的比较方法, 如通过 迭代进行序列数据库检索的 psi-BLAST 方法 ${ }^{[21]}$, 以 及前面提到的用“threading”进行折叠识别 ${ }^{[17]}$ 的方法 等. 最后, 在通过实验或预测确立了目标分子空间结 构模型的前提下, 也可以尝试利用局部空间结构比 较进行功能预测. 例如, 像 SMAP 这类的活性位点比 较方法, 理论上可能用于发现目标蛋白是否存在与 数据库中已有结构相似的活性位点. 本研究组 ${ }^{[22]}$ 近 期报道过一种基于锌离子结合蛋白配位模板的局部 结构比较方法, 可以用于预测给定蛋白质结构中是 否有潜在锌离子结合位点, 以及可能参与锌离子配 位的氨基酸残基.

\section{3 生物分子设计}

序列/结构比较的目标是总结和发现, 而分子设 计的目标是构建和创造 ${ }^{[23]}$. 对蛋白质和核酸等生物 大分子而言, 分子设计即根据期望的结构 (和功能) 设 计序列 ${ }^{[23]}$, 其所针对的是结构功能预测的逆问题. 为 了推动设计方法的发展, 追求合理设计、从头设计是 十分重要的. 在实际应用中, 合理设计与实验室篮 选/定向进化的结合则逐渐成为一种比单纯的进化篮 选更为强大、更为高效的手段 ${ }^{[24 ~ 26] . ~}$

（1）进化设计. 前述序列/结构比较方法所获得 的特定同源家族或特定序列/结构类型的信息, 可用 于指导序列设计. 例如, 在建立了大量与设计目标相 关、相互相似的已知蛋白质分子或核酸分子的多序列 比对后, 可以把多序列比对信息缩略为类似于位置特 异计分矩阵(position-specific scoring matrix, PSSM) ${ }^{[27]}$ 这样的反映不同类型氨基酸(或碱基)在不同位置的 出现频度的简单形式, 并据此设计序列或序列文库. 例如, 在较为保守的位置设计共识(consensus)残基类 型, 在其他位置设计可在一定范围内(变化的残基类 型如疏水类、芳香类、带电类)等. 密歇根大学的 Mitra 等人 ${ }^{[28}$ 提出的蛋白质进化设计方案实际上就是这类 方法, 只是他们使用了非常灵敏的序列比对和折叠 识别方法, 以构建较普适的 PSSM.
PSSM 或类似模型的一大局限是忽略了不同位 置的残基类型间可能存在的相关关系. 如果两个位 置间存在强的残基间相互作用, 考虑这一点对对合 理设计尤为重要. 当用于多序列比对的同源序列足 够多时, 共进化(coevolution) 分析 ${ }^{[29]}$ 可以提供这方面 的信息. Skerker 等人 ${ }^{[30]}$ 通过分析细菌双组分系统中 组氨酸激酶和下游对应响应蛋白的序列共进化, 总 结了二者界面氨基酸的相互依赖关系, 据此设计并 验证了具有新的相互作用特异性、从而能够重新连接 信号传导途径的人工界面. 进化设计的另一策略是 对特性相近但又明确不同的两类生物序列进行比较. 例如, 对同源但具有不同底物(或辅因子)特异性酶的 序列进行比较, 可提示如何设计或改造酶的底物(辅 因子)特异性. 这一方法可进一步推广到仅对活性中 心进行局部比较(不依赖于整体序列结构相似性或同 源性). 本研究组曾用这一策略比较并总结了蛋白质 中还原型辅酶 I (还原型烟酰胺腺嘌呤二核苷酸 (reduced form nicotinamide adenine dinucleotide, $\mathrm{NADH})$ ) 和还原型辅酶 II (还原型烟酰胺腺嘌呤二核 苷酸磷酸(reduced form nicotinamide adenine dinucleotide phosphate, NADPH)) 结合环境的序列差异, 据 此提出的序列改造方案能够以相对较高的成功率 把一个 NADH 特异性结合位点变为 NADPH 特异性 位点.

（2）基于一般性原理的设计. 这类设计所依据 的序列-结构(-功能)关系具有一定普遍性, 而不是仅 适用于属于特定类型或特定同源家族的序列. 最典 型的蛋白质从头设计问题是通过人工设计蛋白质的 氨基酸序列, 让其稳定形成特定的空间结构 ${ }^{[31,32]}$. 目 前蛋白质结构数据库中已有十几个这样人工蛋白质 结构. 其中一部分是螺旋束等特定超二级结构 motif, 其序列是根据经验规则手工设计及再加上实验篮选 优化获得的. 另一部分则用优化序列能量的方法自 动计算设计. 目前真正实验验证成功过的蛋白质序 列自动计算设计方法仅限于加州理工学院 Mayo 研究 组 ${ }^{[33]}$ 的 ORBIT 和华盛顿大学 Baker 研究组 ${ }^{[34]}$ 的 RosettaDesign, 二者采用的能量模型非常接近, 实际 设计的成功率都还很低. 本研究组 ${ }^{[35]}$ 最近建立了一 种新的统计能量模型, 理论验证表明其和 RosettaDesign 在设计空间和设计成功率方面能够互补和竞 争. 用该模型获得了 4 条实验证明能够稳定折叠的人 工蛋白序列(其中 3 条包含实验笁选的点突变), 已解 
析其中两种人工设计蛋白质溶液构象, 证明其与设 计目标高度一致.

蛋白质序列设计可用于设计自然界不存在的人 工酶或人工的分子间相互作用. 近年来, 这两方面均 有成功的例子报道(主要来自华盛顿大学 Baker 研究 组及其合作者 $)^{[36,37]}$. 由于目前设计所使用的模型仍 然包含大量假设, 设计还难以做到百分之百成功, 少 数具有预期功能的设计结果初始活性也较低. 然而, 在这些例子中, 合理设计已经足以为实验篮选提 供一个足够小但又包含正向结果的候选方案集合, 或者提供能够以其为起点进行定向进化优化的初始 活性.

\section{2 网络层次的计算与设计}

\section{1 网络动力学模型}

在合成生物学中, 通过对网络进行动力学分析, 可以预测网络是否具有预期的动力学调控特性, 进 而设计具有特定信息处理能力的人工网络 ${ }^{[38]}$. 这一 方面可以帮助人们更好地理解实际生物网络的设计 原理, 另一方面为通过人工调控控制细胞打下基础.

(1) 模型建立. 在生化网络层次的动力学模型中, 一般用各种生物分子数量(浓度)随时间的变化来刻 画细胞状态的动力学变化 ${ }^{[39]}$. 建立这类模型的第一 步是确定状态变量. 状态变量除了对应于直接感兴 趣的生物分子(如某种蛋白质)的数量或浓度的数量 外, 还包括其他可能影响和调节这些分子的相关分 子(如 mRNA、转录因子等)的数量和浓度. 下一步是 要确定用来模拟不同分子之间相互影响、调节过程的 动力学方程, 如各状态变量如何随时间变化的常微 分方程(ordinary differential equation, ODE)模型. 可 以根据这些过程的生化特性来选择动力学方程所具 有的数学形式: 例如, 用 $x$ 代表苂光蛋白浓度, 其受 诱导物促进的表达过程和后续降解过程可能用如下

的 ODE 模型模拟: $\frac{\mathrm{d} x}{\mathrm{~d} t}=k_{+}+\frac{m k_{+}\left[\frac{\alpha}{K_{a}}\right]^{n}}{1+\left[\frac{\alpha}{K_{a}}\right]^{n}}-k_{-} x$. 接下

来, 要逐一考察模型中所包含的参数和环境变量, 例 如, 在模型中包含了动力学参数 $k_{+}, m, k_{-}$等, 分别代 表 $x$ 的本底表达速率、表达速率增加的最大倍数、降
解速率等. $K_{\alpha}$ 和 $m$ 则分别是反应对诱导过程对诱导物 浓度的敏感性和协同性的参数. 如果可能影响诱导 物浓度 $\alpha$ 的过程已经被包含在模型中了, $\alpha$ 是状态变 量; 否则 $\alpha$ 是环境变量. 最后要考虑如何选择模型中 的参数和环境变量. 这首先取决于建模立模型的主 要目的, 其次取决于对实际体系认识了解的程度. 在 某些情况下, 模型的主要目的是从定量角度再现实 际体系的行为, 此时要求对实际体系应有足够充分 的了解, 从而能够对模型中的动力学参数和环境变 量做出足够精确的估计, 才可能保证预测结果有定 量价值. 在更多的情况下, 不试图通过模型做出定量 的预测, 而是要界定实际体系的动力学特性(如是否 有单一或多个稳定状态, 是否可能产生周期性振荡 等), 分析特定动力学特性的产生机制及其对与参数 或环境变量的依赖关系等. 这类分析有可能在不指 定某些参数的情况下通过解析求解完成, 更多情况 下可能需要采用对某些参数(或参数组合)的遍历或 抽样等计算手段来完成.

(2) 数值模拟与解析分析. 对于 ODE 形式的动 力学模型, 在给定初始条件后, 可以采用数值模拟获 得状态变量随时间演化的轨迹, 从而发现体系是否 存在动力学稳态 (steady state) 或极限环 (limiting cycle) ${ }^{[40]}$. 在动力学稳态处, 体系的状态 (各状态变量 的值)不再随时间变化; 在极限环上, 体系状态随时 间周期性变化 (状态变量的值按固定周期振荡 $)^{[41]}$. 在 模型较为简单的情况下, 这些分析也可以用解析方 法完成. 解析分析往往更有利于阐明动力学行为的 产生机制, 因此实际研究中针对同一问题, 可用较为 细致、复杂的模型进行数值模拟, 以尽可能保持模型 与真实体系的一致性, 同时(基于数值模拟结果)将模 型概略化、简化后进行解析分析, 以获得对机制和因 果联系更清晰的理解.

(3) 随机模拟 ${ }^{[42]}$. 采用含有随机项的微分方程 模型, 可以模拟分析分子数目随机涨落所产生的噪 声对网络的动力学特性的影响. 参与细胞内生化反 应网络的分子数目差异很大, 从数个到数千个不等. 对分子数量较多的体系, 分子数的相对涨落较小, 确 定性 ODE 方程与随机模拟的平均结果应趋于一致. 在分子数较少的情况下, 随机涨落可能导致体系的 实际行为偏离确定性 ODE 模型的预测结果, 此时需 要在模型中考虑随机性. 以经典的基因拨动式开关 
(toggle switch) 为例, 这是最早人工设计并实验验证 的具有双稳态的基因线路. 发现在参与相互抑制的 两种转录因子分子数较少的情况下, 确定性方程所 预测的体系稳态特性和实验观察并不一致; 模拟分 析表明在特定条件下随机涨落可能导致体系相空间 中出现新的中间稳态, 这与实验观察结果一致 ${ }^{[43]}$.

\section{2 代谢网络模型}

（1）流量平衡分析. 流量平衡分析(flux balance analysis, FBA)利用物质守恒的基本原理和中间物含 量处于稳态的基本假定, 建立对各代谢反应流量的 约束条件 ${ }^{[44]}$. 每一种代谢中间物的稳态条件对应于 一个关于该中间物所参与的所有代谢反应流量的线 性方程. 潜在可能的代谢流量组合要满足所有约束 方程, 因此远少于流量的任意组合. 借助随机抽样、 线性规划等数学工具, 可以分析在 FBA 约束下, 体 系能够实现的“最强”代谢能力, 例如, 积累生物量的 最大速率, 产生某种代谢产物的最快速率等. 在合成 生物学中, 可以在对代谢网络进行实验改造之前, 用 FBA 分析比较不同的理论改造方案, 选择、总结出最 佳方案 ${ }^{[45]}$. FBA 分析还可以和同位素标记碳源的核 磁共振(nuclear magnetic resonance, NMR)实验、质谱 实验等结合, 分析确定实际细胞代谢网络中的代谢 流量, 为代谢网络的设计和改造提供指导.

(2) 代谢途径设计. 合成生物学的研究目标往 往涉及把不同物种来源的代谢反应整合起来, 形成 新的人工生物合成途径, 以利用特定原料生产目标 产物. 计算生物学可用于从代谢反应数据库中包含 的大量代谢反应提取、整理出能够完成预期转化任务 的代谢途径 ${ }^{[46]}$. 代谢途径设计工具的发展相对较为 滞后. 目前的计算方法还仅是根据数据库中已有反 应的反应物和产物进行精确匹配检索, 如果原料或
目标产物在数据库中不存在, 或者某步中间反应在 数据中不存在, 计算不可能返回结果. 现有工具还不 能根据数据库中反应物/产物的类似性或反应机理的 相似性提出或建立代谢途径.

\section{3 总结与展望}

合成生物学在方法学上的目标是工程化设计改 造生命体系, 它比传统生物工程更紧密依赖于计算 和设计. 计算科学方法应用于生物学问题研究已经 有三四十年的历史, 在近 10 年内发展特别快速. 在 应用于基因组、转录组等高通量组学数据分析之外, 计算生物学还在生物分子序列结构研究、调控网络分 析、代谢网络分析等方面建立了一系列丰富、有效的 研究手段和方法, 可以应用于分子水平的元器件设 计与优化、调控线路设计、代谢网络设计优化等合成 生物学问题. 本文对有关方法的总结和介绍显然受 到作者本人研究背景和水平限制, 难以真正做到系 统全面. 基于这些有限的讨论可以确定的是, 合成生 物学为生物学计算和实验研究之间的紧密结合提供 了独特的条件和发展机会. (i) 在合成生物学研究 中, 越来越多的计算设计方法更客观、更直接地面临 实验检验; (ii) 越来越多的作为合成生物学课题提 出的研究任务需同时采用计算设计和实验手段、二者 互相补充才可能完成. 在此过程中, 既有计算设计方 法的可靠程度将会进一步改善, 会被更广泛应用于 不同类型的生物学问题; (iii) 围绕合成生物学研究 中出现的新需求, 将会催生一些新的计算方法, 特别 是能够和实验手段互相配合、达成重要但目前还十分 困难的合成生物学目标的方法, 包括设计能够催化新 化学反应的人工酶和感受物理和化学信号的人工调 控分子、设计能够合成非天然代谢物的代谢途径等.

\section{参考文献}

1 Smith T F, Waterman M S. Identification of common molecular subsequences. J Mol Biol, 1981, 147: 195-197

2 Needleman S B, Wunsch C D. A general method applicable to the search for similarities in the amino acid sequence of two proteins. J Mol Biol, 1970, 48: 443-453

3 Holm L, Sander C. Mapping the protein universe. Science, 1996, 273: 595-603

4 Altschul S F, Gish W, Miller W, et al. Basic local alignment search tool. J Mol Biol, 1990, 215: 403-410

5 Felsenstein J. Evolutionary trees from DNA sequences: a maximum likelihood approach. J Mol Evol, 1981, 17: 368-376

6 Shindyalov I N, Bourne P E. Protein structure alignment by incremental combinatorial extension (CE) of the optimal path. Protein Eng, 1998, 11: 739-747 
7 Stormo G D. DNA binding sites: representation and discovery. Bioinformatics, 2000, 16: 16-23

8 Lawrence C E, Altschul S F, Boguski M S, et al. Detecting subtle sequence signals: a Gibbs sampling strategy for multiple alignment. Science, 1993, 262: 208-214

9 Bromley E H, Channon K, Moutevelis E, et al. Peptide and protein building blocks for synthetic biology: from programming biomolecules to self-organized biomolecular systems. ACS Chem Biol, 2008, 3: 38-50

10 Xie L, Xie L, Bourne P E. A unified statistical model to support local sequence order independent similarity searching for ligand-binding sites and its application to genome-based drug discovery. Bioinformatics, 2009, 25: i305-i312

11 Martin Y C, Kofron J L, Traphagen L M. Do structurally similar molecules have similar biological activity? J Med Chem, 2002, 45: $4350-4358$

12 Maggiora G, Vogt M, Stumpfe D, et al. Molecular similarity in medicinal chemistry. J Med Chem, 2014, 57: 3186-3204

13 Merlot C, Domine D, Cleva C, et al. Chemical substructures in drug discovery. Drug Discov Today, 2003, 8: 594-602

14 Kryshtafovych A, Fidelis K, Moult J. CASP10 results compared to those of previous CASP experiments. Proteins, 2014, 82 Suppl 2: $164-174$

15 Marti-renom M A, Stuart A C, Fiser A, et al. Comparative protein structure modeling of genes and genomes. Annu Rev Biophys Biomol Struct, 2000, 29: 291-325

16 Jones D T, Taylor W R, Thornton J M. A new approach to protein fold recognition. Nature, 1992, 358: 86-89

17 Peng J, Xu J. Low-homology protein threading. Bioinformatics, 2010, 26: i294-i300

18 Mathews D H. Revolutions in RNA secondary structure prediction. J Mol Biol, 2006, 359: 526-532

19 Zuker M. Mfold web server for nucleic acid folding and hybridization prediction. Nucleic Acids Res, 2003, 31: 3406-3415

20 Pearson W R, Lipman D J. Improved tools for biological sequence comparison. Proc Natl Acad Sci USA, 1988, 85: 2444-2448

21 Altschul S F, Madden T L, Schaffer A A, et al. Gapped BLAST and PSI-BLAST: a new generation of protein database search programs. Nucleic Acids Res, 1997, 25: 3389-3402

22 Zhao W, Xu M, Liang Z, et al. Structure-based de novo prediction of zinc-binding sites in proteins of unknown function. Bioinformatics, 2011, 27: 1262-1268

23 Khoury G A, Smadbeck J, Kieslich C A, et al. Protein folding and de novo protein design for biotechnological applications. Trends Biotechnol, 2014, 32: 99-109

24 Silberg J J, Nguyen P Q, Stevenson T. Computational design of chimeric protein libraries for directed evolution. Methods Mol Biol, 2010, 673: $175-188$

25 Jackel C, Kast P, Hilvert D. Protein design by directed evolution. Annu Rev Biophys, 2008, 37: 153-173

26 Lutz S. Beyond directed evolution—semi-rational protein engineering and design. Curr Opin Biotechnol, 2010, 21: 734-743

27 Sinha S. On counting position weight matrix matches in a sequence, with application to discriminative motif finding. Bioinformatics, 2006, 22: e454-e463

28 Mitra P, Shultis D, Brender J R, et al. An evolution-based approach to de novo protein design and case study on Mycobacterium tuberculosis PLoS Comput Biol, 2013, 9: e1003298

29 de juan D, Pazos F, Valencia A. Emerging methods in protein co-evolution. Nat Rev Genet, 2013, 14: 249-261

30 Skerker J M, Perchuk B S, Siryaporn A, et al. Rewiring the specificity of two-component signal transduction systems. Cell, 2008, 133: 1043-1054

31 Kuhlman B, Dantas G, Ireton G C, et al. Design of a novel globular protein fold with atomic-level accuracy. Science, 2003, 302: 1364-1368

32 Dahiyat B I, Mayo S L. De novo protein design: fully automated sequence selection. Science, 1997, 278: 82-87

33 Dahiyat B I, Sarisky C A, Mayo S L. De novo protein design: towards fully automated sequence selection. J Mol Biol, 1997, 273: 789-796

34 Leaver-Fay A, Tyka M, Lewis S M, et al. ROSETTA3: an object-oriented software suite for the simulation and design of macromolecules. Methods Enzymol, 2011, 487: 545-574

35 Xiong P, Wang M, Zhou X, et al. Protein design with a comprehensive statistical energy function and boosted by experimental selection for foldability. Nat Commun, 2014, 5: 5330

36 Fleishman S J, Whitehead T A, Ekiert D C, et al. Computational design of proteins targeting the conserved stem region of influenza hemagglutinin. Science, 2011, 332: 816-821

37 Siegel J B, Zanghellini A, Lovick H M, et al. Computational design of an enzyme catalyst for a stereoselective bimolecular Diels-Alder reaction. Science, 2010, 329: 309-313

38 Marguet P, Balagadde F, Tan C, et al. Biology by design: reduction and synthesis of cellular components and behaviour. J R Soc Interface, 
2007, 4: 607-623

39 Tyson J J, Novak B. Functional motifs in biochemical reaction networks. Annu Rev Phys Chem, 2010, 61: 219-240

40 Tyson J J, Chen K C, Novak B. Sniffers, buzzers, toggles and blinkers: dynamics of regulatory and signaling pathways in the cell. Curr Opin Cell Biol, 2003, 15: 221-231

41 Goldbeter A. Computational approaches to cellular rhythms. Nature, 2002, 420: 238-245

42 Stewart-Ornstein J, El-Samad H. Stochastic modeling of cellular networks. Methods Cell Biol, 2012, 110: 111-137

43 Ma R, Wang J, Hou Z, et al. Small-number effects: a third stable state in a genetic bistable toggle switch. Phys Rev Lett, 2012, 109: 248107

44 Orth J D, Thiele I, Palsson B O. What is flux balance analysis? Nat Biotechnol, 2010, 28: 245-248

45 Ranganathan S, Suthers P F, Maranas C D. OptForce: an optimization procedure for identifying all genetic manipulations leading to targeted overproductions. PLoS Comput Biol, 2010, 6: e1000744

46 Moura M, Broadbelt L, Tyo K. Computational tools for guided discovery and engineering of metabolic pathways. Methods Mol Biol, 2013, 985: $123-147$

\title{
Computation and Design of Biological Systems at Different Levels in Synthetic Biology
}

\author{
LIU HaiYan \& HUANG Bin \\ School of Life Sciences, University of Science and Technology of China, Hefei 230027, China
}

\begin{abstract}
A central goal of synthetic biology is to integrate the principles and approaches of modern engineering sciences into the engineering of biological systems. The functioning of living systems spans different levels, ranging from molecules to cells and, eventually, the organism. Synthetic biology studies cover the same range, including the design and standardization of molecular parts and devices at the molecular level and the study of design principles of cellular networks by using synthetic gene circuits or the design and implementation of artificial metabolic pathways for biosynthesis at the network level. We briefly introduce related computational approaches applicable at these two levels. In our discussions, we attempt to explore the means by which existing computational approaches may be applied in synthetic biology and elucidate the potential developments in computational tools that would improve them such that they meet the needs of synthetic biology studies.
\end{abstract}

bioinformatics methods, molecular design, network dynamics, metabolomics networks

doi: 10.1360/N052014-00261 